International Journal of Electronics and

Communication Engineering (IJECE)

ISSN(P): 2278-9901; ISSN(E): 2278-991X

Vol. 4, Issue 6, Oct - Nov 2015, 9-16

(C) IASET

\title{
STUDY OF CHIRPED PULSE COMPRESSION IN OPTICAL FIBER FOR \\ ALL FIBER CPA SYSTEM
}

ARPAN DUTTA

Department of Physics, University of Burdwan, Burdwan, West Bengal, India

\begin{abstract}
Fiber lasers are highly regarded in present laser technological fields due to their high efficiency, beam quality and easy thermal management. Although, performance of fiber laser system is challenged by different nonlinear effects at high power. In case of ultra-short high power pulse fiber laser, nonlinearity plays significant role in pulse propagation through optical fiber as a result of presence of the high peak power of short pulses. Chirped pulse amplification (CPA) system can be incorporated in the design of such fiber laser as a way of increasing the pulse energy while mitigating the nonlinear effects. CPA system stretches the chirped pulses coming from fiber laser to reduce its peak power and then linearly amplified it. Amplified pulse experienced a pulse compressor made by bulk diffraction grating employed for recompression of the pulse. This bulk diffraction grating arrangement can be supplanted by specially design fiber arrangements to design all fiber CPA system using the nature of chirped pulse propagation in negative dispersion (anomalous) regime. In this paper, ultra-short chirped pulse propagation and compression in anomalous regime under the influence of Kerr nonlinearity, initial chirp and dispersion is studied using the nonlinear Schrödinger equation for optical fibers for all fiber CPA system.
\end{abstract}

KEYWORDS: Anomalous Dispersion, Chirped Pulse Amplification, Kerr Effect, Nonlinear Schrödinger Equation, Self Phase Modulation

\section{INTRODUCTION}

Fiber lasers are become an essential part of present laser technologies due to their increasing popularity in various application fields. Efficient utilization of high power supporting capability of these lasers while maintaining the beam quality, makes them beneficial for industrial, defense and scientific applications. Although, in spite of all the acclaimed advantages of fiber lasers - stable light guidance over very long distances, large gain, high beam quality, alignment free laser system using narrowband reflectors (known as Fiber Bragg Grating or FBG) directly inside the fiber and combine or split light beams in a single fiber, fiber nonlinearities limit performance of fiber laser systems at high power. Due to the long path lengths of tightly confined light in the fiber core, the perfect conditions are created for inducing non linear effects even at modest power [1].

When high intensity light propagates through the core of the fiber, its refractive index (R.I.) profile is modified in a nonlinear way. The nonlinear dependence of the R.I. of a material on the intensity of light is produced by the Kerr effect [1]. This effect modifies the phase of a light through the nonlinear variation of the optical path. This effect has no significant role on continuous wave $(\mathrm{CW})$ operation but for pulsed operation, Kerr effect produces an instantaneous phase shift which varies across the duration of the pulse. This effect is known as self phase modulation (SPM). In SPM effect, 
new frequency components are generated continuously as the pulse propagates through the fiber. These SPM generated frequency components broaden the spectrum over its initial width. Chirp is the instantaneous frequency change in the pulse envelope. The chirp induced by SPM increases in magnitude with propagated distance and deviate the shape of the spectrum [2]. Ultra short pulse (generally in pico or femto second order) generation using pulsed operation of high power ultrafast fiber lasers yields shorter pulses with very high peak power. Due to high peak power of these pulses, nonlinear effects play significant role in fiber laser performance.

Chirped pulse amplification (CPA) technique can be employed in high power ultrafast fiber lasers to increase pulse energy while mitigating the nonlinear effects. CPA can be used to prevent nonlinear effects in the fiber laser and amplifiers to enhance spectral quality of the pulse with pulse energies in order of micro joule to milijoule. CPA can generate sub picoseconds pulses with pulse energies of around 1 micro joule which are useful for many applications [3].

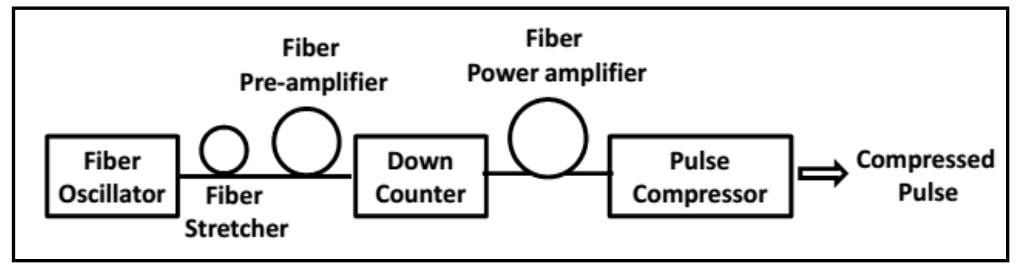

Figure 1: Chirped Pulse Amplification (CPA) System

The working principle of CPA system can be understood from the block diagram given in figure 1. Fiber oscillator which is typically a mode locked fiber laser act as a short pulse source and deliver sub picoseconds pulses. These short pulses are stretched by fiber stretcher, using the chromatic dispersion in several hundred meters of single mode fiber. Consequently, the peak power of the pulses is greatly reduced due to stretching and hence the nonlinear effects are mitigated. These stretched pulses are linearly amplified by the fiber power amplifier. Amplified pulses are passes through a pulse compressor arrangement which can be made by a pair of bulk diffraction grating, inserted at the output of the power amplifier to reverse the chromatic dispersion induced by the stretcher and to recompress the pulses. The optical down converter is inserted to reduce the repetition rate of the oscillator pulses and thus to increase the pulse energy. By implementing the CPA system with current fiber technology, pulse stretching and recompression up to a factor 10000, pulse energies of several mille joules and average powers of up to $1 \mathrm{~kW}$ can be achieved [3].

To design an all fiber CPA system, all the main components of the CPA system should be based on optical fibers. In CPA system, oscillator, stretcher and power amplifier can be made by using optical fibers. If the use of bulk diffraction grating in compressor part replaced by specially designed fiber arrangements then all fiber CPA system can be achieved [4]. Now, pulse propagation in optical fiber mainly affected by the group velocity dispersion and fiber nonlinearity. Pulse, with presence or absence of chirp, propagating in nonlinear dispersive medium, experienced different effects at different propagation regime. Nonlinear effect like self phase modulation affects the pulse in spectral domain where as dispersion affect the pulse in temporal domain. Compression of positively chirped pulses also happens in negative dispersion (anomalous) regime [5]. So replacement of bulk diffraction grating in compressor part can be possible if the desired compression of pulse obtained by controlling the pulse as well as the optical fiber parameters.

\section{Chirped Pulse Propagation in Anomalous Regime}

Nonlinear Schrödinger equation (NLSE) governs the propagation of optical pulses in nonlinear dispersive medium 
[2]. It is mathematically expressed as

$$
i \frac{\partial A}{\partial z}=-\frac{i \alpha}{2} A+\frac{\beta_{2}}{2} \frac{\partial^{2} A}{\partial T^{2}}-\gamma|A|^{2} A
$$

Where $A(z, T)=$ slowly varying amplitude of the pulse envelope, $\alpha=$ fiber loss, $\beta_{2}=2^{\text {nd }}$ order dispersion, $\gamma=$ nonlinear coefficient.

The three terms on the right hand side of equation (1.0) signifies, respectively, the effects of fiber loss, dispersion and nonlinearity on pulses propagating inside the fibers. Depending upon the incident pulse parameters - peak power $\left(\mathrm{P}_{0}\right)$ and initial width $\left(\mathrm{T}_{0}\right)$ of the pulses, either dispersive or nonlinear effects dominate along the fiber. The values of dispersion length $\left(\mathrm{L}_{\mathrm{D}}\right)$ and nonlinear length $\left(\mathrm{L}_{\mathrm{NL}}\right)$ of the fiber provide length scaling over which dispersive or nonlinear effects become important for pulse evolution. Mathematically

$$
L_{D}=\frac{T_{0}^{2}}{\left|\beta_{2}\right|} \quad L_{N L}=\frac{1}{\gamma P_{0}} .
$$

The sign of $\beta_{2}(=\mathrm{s})$ implies two different dispersion regime in optical fiber. Positive value of $\mathrm{s}$ implies normal or positive dispersion regime where as negative value of $s$ indicates the anomalous or negative dispersion regime. Pulse propagating in fiber experienced different effects in these two regimes. Again, Chirped and unchirped pulses also have different experience during propagation in these two regimes.

Consider a linearly chirped pulse with Gaussian profile which is represented as

$$
A(0, T)=\exp \left[-\frac{(1+i C)}{2} \frac{T^{2}}{T_{0}^{2}}\right]
$$

Where $\mathrm{C}$ is chirp parameter. If instantaneous frequency increases linearly from leading to trailing edge of the pulse then it is up chirp $(\mathrm{C}>0)$ and if opposite occurs then it is down chirp $(\mathrm{C}<0)$.

The spectrum of $\mathrm{A}(0, \mathrm{~T})$ can be written as

$$
A(0, \omega)=\left(\frac{2 \pi T_{0}^{2}}{1+i C}\right)^{1 / 2} \exp \left[-\frac{\omega^{2} T_{0}^{2}}{2(1+i C)}\right]
$$

The full width half maximum (FWHM) value in spectral domain is

$$
\Delta \omega=\left(1+C^{2}\right)^{1 / 2} / T_{0}
$$

In absence of chirp $(\mathrm{C}=0)$, spectral width is transform limited $\left(\Delta \omega T_{0}=1\right)$ i.e. maintaining the time bandwidth product [6]. So presence of chirp enhance the spectral width of a pulse by factor of $\left(1+C^{2}\right)^{1 / 2}$ and equation (1.3) can be used to estimate the value of $\mathrm{C}$.

The transmitted pulse at any distance $\mathrm{z}$ is 


$$
A(z, T)=\frac{T_{0}}{\left[T_{0}^{2}-i \beta_{2} z(1+i C)\right]^{1 / 2}} \exp \left(-\frac{(1+i C) T^{2}}{2\left[T_{0}^{2}-i \beta_{2} z(1+i C)\right]}\right)
$$

So a chirped Gaussian pulse also maintains its shape during propagation. The pulse width after propagating a distance $\mathrm{z}$ is

$$
\frac{T_{1}}{T_{0}}=\left[\left(1+\frac{C \beta_{2} z}{T_{0}^{2}}\right)^{2}+\left(\frac{\beta_{2} z}{T_{0}^{2}}\right)^{2}\right]^{1 / 2}
$$

The chirp parameter of the pulse changes as

$$
C_{1}(z)=C+\left(1+C^{2}\right)\left(\beta_{2} z / T_{0}^{2}\right)
$$

Chirped pulses may broaden or compress depending upon whether $\mathrm{C}$ and $\beta_{2}$ have same sign or not.

If $\beta_{2} \mathrm{C}>0$, the chirped Gaussian pulses are broaden monotonically. This is because the dispersion induced chirp adds to the input chirp as the two contributions have same sign.

If $\beta_{2} \mathrm{C}<0$, the dispersion induced chirp is opposite of input chirp. So pulse width decreases and minimum at a distance. The minimum value of the pulse width depends on input chirp parameter as

$$
T_{1}^{\min }=\frac{T_{0}}{\left(1+C^{2}\right)^{1 / 2}}
$$

When the pulse attains its minimum width, $\mathrm{C}_{1}=0$ and pulse becomes transform limited such that $\Delta \omega_{0} T_{1}^{\text {min }}=1$ where $\Delta \omega_{0}$ is the input spectral width of the pulse.

So if a positively chirped $(\mathrm{C}>0)$ Gaussian pulse propagated through anomalous dispersion regime, then $\beta_{2} \mathrm{C}<0$ and hence pulse compression occurs. If it is possible to control the amount of compression by controlling the pulse and fiber parameters, then the required pulse compressor can be designed.

\section{Simulation Results}

The effects of group velocity dispersion and SPM on pulse propagation in optical fiber can be realized by numerically solving the normalized form of NLSE. The standard numerical code for numerical solution of normalized NLSE written by G.P. Agrawal is used in this paper to study the chirped pulse compression in anomalous regime. The code implement split step Fourier method for solving the equation using MATLAB [2]. The normalized NLSE expressed as

$$
\frac{\partial A}{\partial \xi}=-\frac{i s}{2} \frac{\partial^{2} A}{\partial \tau^{2}}+i N^{2}|A|^{2} A
$$

Where A $(\xi, \tau)=$ slowly varying amplitude of the pulse envelope, normalized distance $(\xi)=\mathrm{z} / \mathrm{L}_{\mathrm{D}}$, normalized time $\tau=\mathrm{T} / \mathrm{T}_{0}$ and $\mathrm{N}^{2}=\mathrm{L}_{\mathrm{D}} / \mathrm{L}_{\mathrm{NL}} \mathrm{N}$ is actually related to the fiber and pulse parameters as 


$$
N=\sqrt{\left(\gamma P_{0} T_{0}^{2} /\left|\beta_{2}\right|\right)}
$$

$\mathrm{s}=\operatorname{sign}$ of $\beta_{2}= \pm 1$ (1 for normal regime and -1 for anomalous regime $)$

In the numerical code, total fiber length is defined in units of the $\mathrm{L}_{\mathrm{D}} \mathrm{N}$ and $\mathrm{s}$ should be specified together with input amplitude $\mathrm{A}(0, \tau)$ for any numerical solution of equation (1.8). The input amplitude is related to the shape and amount of chirp of the pulse as

$$
A(0, \tau)=f(\tau) \exp \left(-i C \tau^{2} / 2\right)
$$

Where $f(\tau)$ represents the pulse shape. In the code, an integer $m$ is used to define the pulse shape. For Gaussian profile, the value of $m=1$. For $m=1$

$$
A(0, \tau)=\exp \left[-\frac{1}{2}(1+i C) \tau^{2}\right]
$$

Now, pulse compression can be achieved in anomalous regime if the pulse is initially positively chirped. Compression can be controlled using the chirp parameter of the pulse. In numerical code, by setting $\mathrm{s}=-1$ (anomalous dispersion regime), $\mathrm{m}=1$ (Gaussian pulse) and $\mathrm{N}=1$, pulse propagation examined by assigning the chirp parameter $0.5,1$ and 2 and varying the fiber length from 0.1 to 0.5 by 0.1 steps (in units of the dispersion length). Observations of the pulse propagation are done in temporal and spectrum domain. In each case, the value of $\mathrm{C}$ implies the initial pulse is positively chirped.

To design an all fiber CPA system, replacement of bulk diffraction grating in the pulse compressor part is only possible if the required compression is achieved by specially designed fiber arrangements. The study in this paper also finds the optimized fiber length with fixed fiber and pulse parameters for required pulse compression.

An experimentally obtained output pulse of a mode locked fiber laser system was taken for this study. Pulse and fiber parameters are - peak power $\left(\mathrm{P}_{0}\right)=55 \mathrm{~W}$, nonlinearity parameter $(\gamma)=3.5 / \mathrm{W} / \mathrm{km}, \mathrm{T}_{\mathrm{FWHM}}=5 \mathrm{ps}, \mathrm{N}=15.5$ and $\left|\beta_{2}\right|=2 \times 10^{-26}$
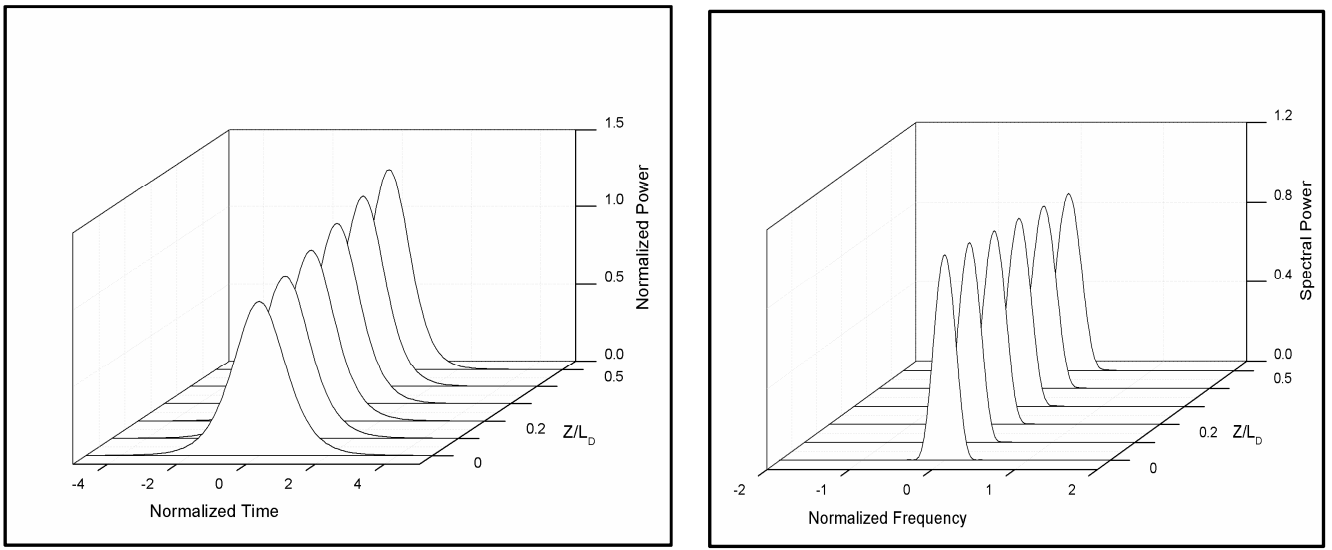

Figure 2: Chirped Pulse Propagation in Anomalous Dispersion Regime with $\mathrm{C}=0.5$ 

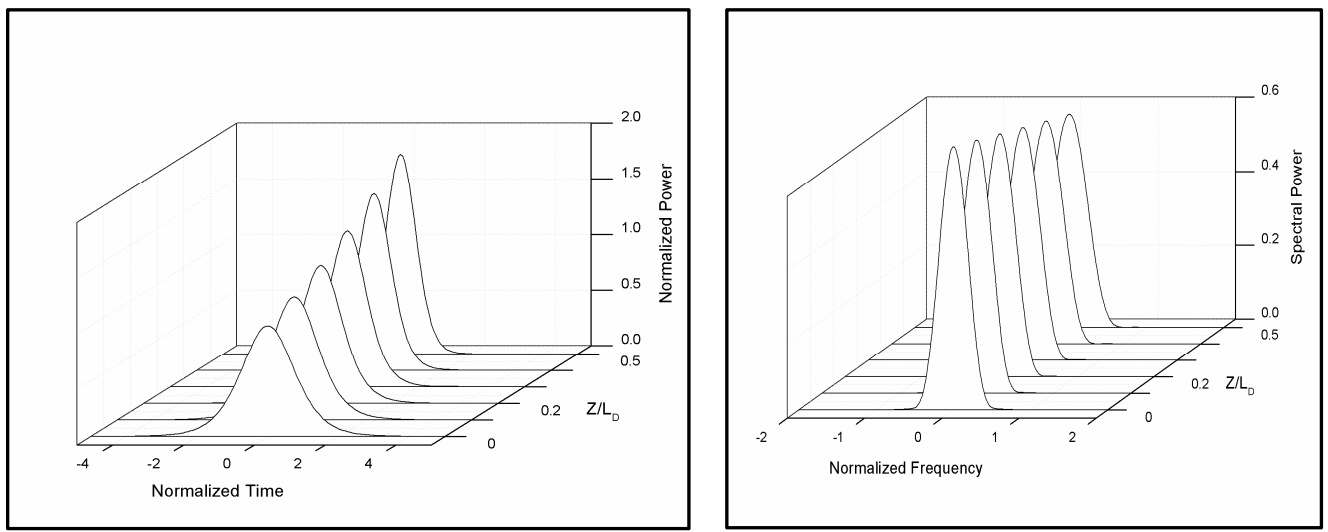

Figure 3: Chirped Pulse Propagation in Anomalous Dispersion Regime with $\mathrm{C}=1$
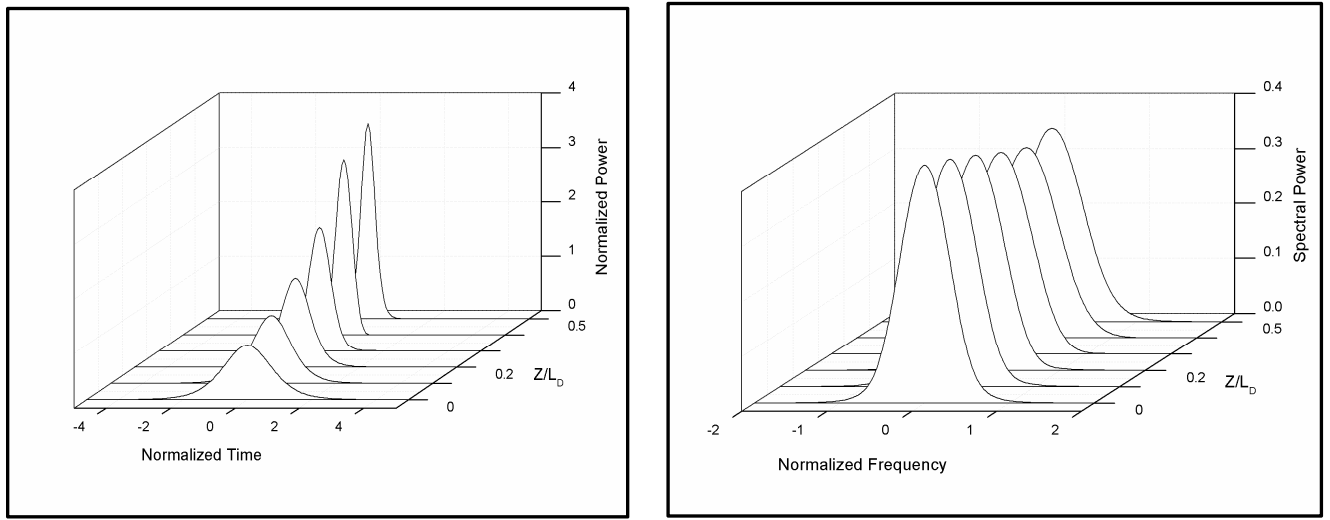

Figure 4: Chirped Pulse Propagation in Anomalous Dispersion Regime with $\mathrm{C}=2$
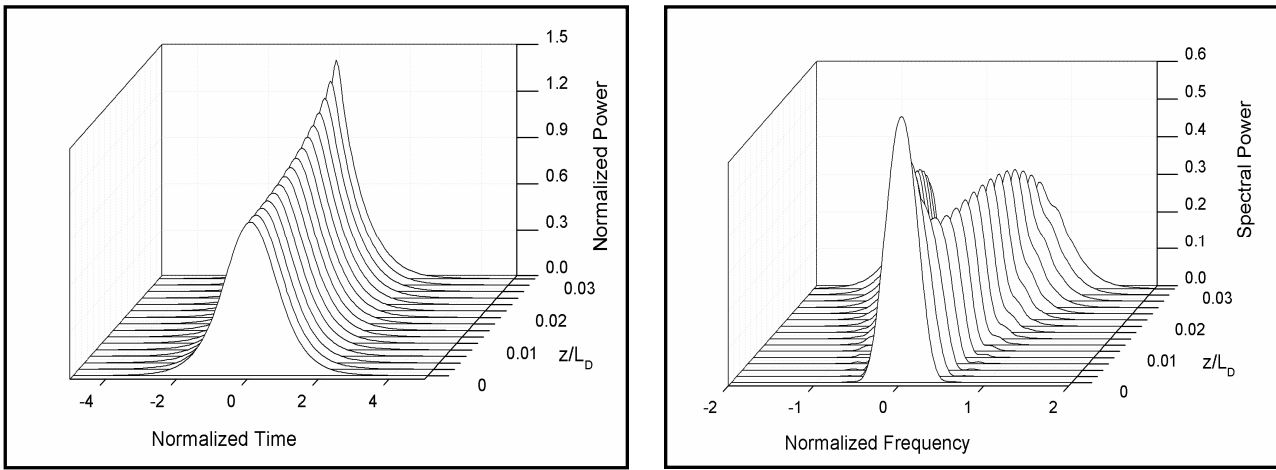

Figure 5: Compression of an Experimentally Obtained Pulse in Anomalous Dispersion Regime

\section{CONCLUSIONS}

According to figure 2 to figure 4, it can be concluded that positively chirped pulse propagation in anomalous regime results pulse compression in temporal domain while no change in spectral domain. In all the three cases i.e. $\mathrm{C}=$ $0.5,1$ and 2 , pulse width reaches its minimum value at $0.5 \mathrm{~L}_{\mathrm{D}}$. When the pulse attains its minimum width, it becomes transform limited. Thus to maintain the transform limit, no change occur in spectral width of the pulse and hence no change occur in spectral domain. Pulse compression i.e. the minimum value of the pulse width depends on the input chirp parameter by equation (1.7). If we move from figure 2 to figure 4 , we can conclude that increment in positive chirp results 
increment in pulse compression in temporal domain but no change in spectral domain. More the input pulse is initially chirped, more pulse compression occurs temporally.

Figure 5 shows the compression of the experimentally obtained pulse. The initial pulse has $\mathrm{T}_{\mathrm{FWHM}}=5 \mathrm{ps}$ before propagation. At fiber length $0.03 \mathrm{~L}_{\mathrm{D}}$, the pulse width becomes minimum and we get compressed pulse with $\mathrm{T}_{\mathrm{FWHM}}=1 \mathrm{ps}$ after propagation. So the key results of this study are

Optimized fiber length $=0.03 \mathrm{~L}_{\mathrm{D}}=37.5 \mathrm{~m}\left(\mathrm{as} \mathrm{L}_{\mathrm{D}}=1.25 \mathrm{~km}\right)$.

$\mathrm{FWHM}$ before propagation $=5 \mathrm{ps}$ and $\mathrm{FWHM}$ after propagation $=1 \mathrm{ps}$.

So Compression factor $=0.2$.

Estimated Chirp parameter using equation (1.7), $\mathrm{C}=4.9$

Unlike the previous results, figure 5 shows a significant change in pulse spectra. The spectrum of the pulse becomes a multi peak structure due to the SPM induced chirp. This happens due to the high value of $\mathrm{N}$. N = 15.5 imply dominant nonlinear effects on the pulse. Higher value of $\mathrm{N}$ can cause optical wave breaking due to high nonlinearity.

So from the overall study we can inferred that pulse compression can be controlled using the initial chirp of the input pulse without affecting the pulse spectra and fiber lengths can be optimized with fixed pulse and fiber parameters for required compression. These important facts lead us to the design of an efficient pulse compressor for all fiber CPA system.

\section{ACKNOWLEDGEMENTS}

I would like to express my heartiest gratitude to Dr. Mrinmay Pal, Senior Scientist, Fiber Optics and Photonics Division (FOPD), CSIR-Central Glass and Ceramic Research Institute (CGCRI), for his valuable guidance and cooperation to complete this work. I am also thankful to the Director of the CSIR-CGCRI for giving me the opportunity to use the CGCRI laboratory and scientific software for my work.

\section{REFERENCES}

1. Cesar Jauregui, Jens Limpert and Andreas Tünnermann, High Power Fiber laser, nature photonics vol.7, nov. 2013,pg 861-867.

2. G. P. Agarwal, Nonlinear Fiber Optics, Academic press.

3. Martin E. Fermann and Ingmar Hartl, ultra fast Fiber lasers, nature photonics vol.7 november 2013, pg868-874.

4. N.G.R. Broderick, D. J. Richardson, D. Taverner, J.E. Caplen, L. Dong, M. Ibsen, “ High Power Chirped Pulse all fiber amplification system based on large mode area fiber components", Optics Letters, Vol.24, Issue 8, 1999, pp. $566-568$.

5. S. V. Smirnov, S. M. Kobtsev and S. V. Kukarin, Linear compression of chirped pulses in optical fiber with large step-index mode area, 23 Feb 2015, Vol. 23, No. 4, Optics Express 2015.

6. Aaron Webster, Useful Mathematical Formulas for Transform Limited Pulses, Dover publications, 1964. 